\title{
El Open Access a debate: entre el pago por publicar y la apertura radical sostenible
}

\author{
Verónica Araiza Díaz* \\ María Esther Ramírez Godoy** \\ Alma Silvia Díaz Escoto**
}

Artículo recibido:

11 de octubre de 2018

Artículo aceptado:

20 de febrero de 2019

Artículo de revisión

\section{Resumen}

Se muestra la evolución del movimiento de acceso abierto, en contraste con la resistencia de empresas editoriales que desean seguir controlando la distribución de la información científica a través del pago por publicar. Se presenta a los actores y el escenario donde se da esta disputa, con base en los antecedentes de la comunicación científica y de las diferentes etapas del proceso de acceso a las publicaciones especializadas. Se discute la relevancia y viabilidad del movimiento Open Access (AO), y se proporcionan algunas claves para su posible sustentabilidad. Se destaca la necesidad de replantear la dictaminación por pares de los artículos, a fin de alcanzar una revisión abierta (Open Review, $\mathrm{OR}$ ), en un contexto generalizado de cultura libre. Se

* Escuela Nacional de Estudios Superiores, Universidad Nacional Autónoma de México, Mérida. México veraiza@yahoo.com

** Dirección General de Bibliotecas, Universidad Nacional Autónoma de México, México estherr@dgb.unam.mx alside@yahoo.com

INVESTIGACIÓN BIBLIOTECOLÓGICA, vol. 33, núm. 80, julio/septiembre, 2019, México, ISSN: 2448-8321 pp. 195-216 
concluye que se trata de un fenómeno de carácter económico, social, político y epistémico, además de que la esencia del Open Access demanda un modelo de circulación libre, abierta y gratuita del conocimiento científico, en un contexto de prácticas colaborativas entre autores, editores, revisores y bibliotecarios, en el entendido de que el conocimiento es un bien común y que, por ello, es necesario definir nuevos patrones de producción, evaluación, distribución, consumo y propiedad del conocimiento.

Palabras clave: Acceso Abierto; Información Científica; Cultura Libre; Gestión de Información.

\author{
Open Access debate: Between article processing \\ charge and radical sustainable openness \\ Maria Esther Ramirez Godoy, Verónica Araiza Diaz and \\ Alma Silvia Díaz Escoto
}

\begin{abstract}
The evolution of the Open Access (OA) movement is shown in contrast to the resistance of commercial enterprises that intend to keep controlling the distribution of scientific information through Article Processing Charge (or pay for publishing). Actors and the scenery in dispute are shown based on the antecedents of scientific communication and the different results of scientific research, as well as the different stages of the process of access to specialized publications. The relevance and viability of the Open Access movement are discussed and some clues or key points are provided for its possible sustainability. The need to emphasize the relevance is placed upon the intention of rethinking the way of assessing by peer-reviewing, in order to achieve an Open Review (OR) in an extended context of free culture. It is concluded that this is an economic, social, political and epistemic phenomenon, and that the essence of the Open Access demands a model that contemplates the open and free circulation of scientific knowledge in a context of collaborative practices among authors, publishers, reviewers, and librarians; with the understanding that knowledge is a common good and that it is therefore necessary to define new patterns of production, evaluation, distribution, consumption, and ownership of knowledge.
\end{abstract}

Key Words: Open Access; Scientific Information; Free Culture; Information Management. 


\section{INTRODUCCIÓN}

A principios del siglo XXI surgió el Open Access (OA) como un mocaciones científicas bajo el control de una pequeña oligarquía académica y unas cuantas empresas proveedoras de información científica (ABC Ciencia, 2015). En los últimos años, se ha vuelto un tema de la mayor relevancia para las bibliotecas universitarias y las comunidades académicas y científicas, las cuales han presionado en todo el mundo para que los resultados de la investigación científica estén disponibles en línea, en texto completo y en acceso abierto para todos los usuarios. Con base en la iniciativa Open Access 2020 (Conferencia de Berlín, 2015), se pretende que todas las publicaciones que resulten de la investigación científica sean abiertas y reutilizables, y que los costos detrás de su difusión sean transparentes y económicamente sostenibles, con el fin de que tengan una mayor difusión en beneficio de los investigadores, el sector de innovación y la sociedad; al mismo tiempo, se busca una mayor visibilidad de los resultados de la investigación. Asimismo, que pueda ofrecerse a las pequeñas y medianas empresas acceso a los últimos resultados de investigación para su aplicación.

Sin duda, se trata de una postura crítica frente al modelo hegemónico de comercialización, difusión y valoración de la información científica, además de que cuestiona las limitaciones geográficas y lingüísticas que impone el esquema dominante y la necesidad de visibilizar la producción científica de todos los países.

En contrapartida, editores y proveedores de información científica se han sumado a esta iniciativa con peculiares propuestas, de tal suerte que han conseguido que el tema del OA se integre a sus esquemas tradicionales lucrativos de difusión científica, por medio de su modelo de pago por publicar artículo por artículo.

No obstante, el esquema alternativo que se propone se basa en la noción de Bienes Comunes de Información (BCI), que supone que la información y el conocimiento no son mercancías; de hecho, se centra en el valor de uso (y no de cambio) de estos recursos que se dice son cruciales para el desarrollo científico, social y humano, con lo cual se quiere limitar el monopolio sobre los recursos de conocimiento. 


\section{Metodología}

Con base en una revisión documental, se muestra la evolución del movimiento que demanda el acceso abierto a la información científica, en contraste con el modelo comercial de suscripción, a través de sus aspectos económicos, sociales, tecnológicos y jurídicos más relevantes. Para facilitar la comprensión del fenómeno, es pertinente presentar ampliamente a los actores y el escenario donde ocurre esta disputa, por lo mismo nos extendemos en la presentación de los antecedentes y las diferentes etapas del proceso de acceso a las publicaciones científicas.

Además, discutimos la relevancia y viabilidad del movimiento del OA y proporcionamos algunas claves para su posible sustentabilidad. Al mismo tiempo, evidenciamos las estrategias empresariales para perpetuar el control sobre la comercialización y evaluación de la información científica. Por último, se presenta un análisis y algunas propuestas.

\section{Pista CONCEPTUAL}

El Acceso Abierto (AA) es una forma de compartir información académica o científica, ya sea original o de divulgación, sin ningún costo o restricción para el usuario. Es un movimiento internacional que se basa en que un autor o titular de los derechos de una obra autorice el libre acceso para usar, distribuir, copiar y transmitir el trabajo públicamente, y de manera gratuita, así como el derecho de hacer un pequeño número de copias para su uso personal, sin pagar derechos de autor, en cualquier medio digital para cualquier finalidad, sujeto a la debida referencia de autoría. Este movimiento sienta sus bases en las Declaraciones de Budapest, Bethesda y Berlín (OSI, 2001; Howard Hughes Medical Institute, 2003; Sociedad Max Planck, 2003).

En este trabajo se consideran dos definiciones de OA:

1) La de la Budapest Open Access Initiative (BOAI) (OSI, 2001), que implica el libre acceso a los textos completos a través de Internet, así como su uso y distribución, con el debido respeto a las leyes de propiedad intelectual existentes, en el entendido de que la boai aboga por que sean los autores o las instituciones quienes dispongan de estos derechos.

2) La de la Declaración de Bethesda (Howard Hughes Medical Institute, 2003) que asume la anterior, pero la complementa con la garantía de que el derecho de autor no sea una barrera para el acceso a los artículos y que los índices de estos pueden almacenarse en repositorios institucionales. 
De acuerdo con la Declaración de Budapest:

Por acceso abierto a esta literatura (científica) queremos decir que está disponible gratis para el público en Internet, se permite a los usuarios su lectura, descarga, copia, distribución, impresión, búsqueda o enlazado a los contenidos completos de estos artículos, recolectarlos para su indexación, pasarlos como datos para software o utilizarlos para cualquier otro propósito legítimo, sin más barreras financieras, legales o técnicas que aquellas que supongan acceder a Internet. La única restricción a la reproducción y distribución, y el único rol para la propiedad intelectual en este dominio, debería ser dar a los autores el control sobre la integridad de su trabajo y el derecho a ser debidamente reconocido y citado (OSI, 2001).

Por su parte, las declaraciones de Bethesda y Berlín señalan que:

Para que un trabajo sea de acceso abierto, el beneficiario del copyright debe consentir, por adelantado, dejar que los usuarios copien, usen, distribuyan, transmitan y visualicen el trabajo públicamente, y hacer y distribuir trabajos derivados, en cualquier otro medio digital, para cualquier propósito responsable, sujeto únicamente a la atribución de la autoría (Howard Hughes Medical Institute, 2003; Sociedad Max Planck, 2003).

Mientras que Peter Suber acota que la literatura científica de acceso abierto:

Es digital, en línea, gratuita y se encuentra [exenta] de la mayoría de derechos de autor y restricciones de licencias. Lo que la hace posible es la Internet y el consentimiento del autor o del titular del copyright. En la mayoría de los campos del conocimiento, las revistas especializadas no pagan a los autores, quienes, por consiguiente, pueden autorizar el acceso abierto, sin que ello repercuta en sus ingresos. El acceso abierto es absolutamente compatible con la revisión por parte de expertos, y la mayoría de iniciativas de acceso abierto destacables en el ámbito de la literatura académica insisten en la importancia de este punto (Suber, 2004).

\section{Antecedentes del Acceso Abierto}

La mayor parte de los resultados de la investigación científica se publicaban como artículos en revistas científicas y académicas en formato impreso, y a partir de 1995 también en formato digital. El modelo de comunicación científica se basa principalmente en dos aspectos: 1) la forma de presentar los resultados de una investigación y 2) la forma de evaluar su impacto en otras investigaciones. 
La medida del impacto de la actividad científica o evaluación se inicia en los años sesenta, con la creación del Institut for Scientific Informatión (ISI) y Thomson Scientific, que desarrolló una serie de índices, como el Science Citation Index (SCI), Social Science Citation Index y Art and Humanities Citation Index, que reúnen los registros de determinadas publicaciones científicas editadas en el mundo, que se seleccionan con criterios muy estrictos, para expresar, junto con la referencia bibliográfica de cada artículo, todas las referencias bibliográficas del propio artículo y las citas que recibe dentro del grupo de revistas que están en la base de datos.

Aunado a esto, se obtienen otra serie de datos que permite evaluar a los autores, las revistas y las instituciones de acuerdo con sus publicaciones en el grupo de revistas indizadas en estos índices. Debe exponerse que la mayoría de estos títulos se publican en inglés, con lo que se ha generado un importante sesgo a favor de la investigación anglosajona. De tal suerte que gran parte de las evaluaciones científicas y de los estudios sobre el uso de información científica realizadas en países de todo el mundo toman como referencia estos índices, y de esta manera se obliga a los investigadores de todo el mundo a dirigir sus esfuerzos y objetivos en la comunicación de sus resultados en las publicaciones incluidas en el ISI.

Por otro lado, a partir de 2010, Elsevier creó la base de datos Scopus, que proporciona facilidades para analizar los resultados de la producción editorial desde diversos enfoques, ya que se recuperan referencias bibliográficas, resúmenes, textos completos, citas, patentes, análisis evaluativos de más de veinte mil títulos; además, permite conocer diferentes aspectos relacionados con las citas a los trabajos publicados. De tal forma que se ha ampliado el espectro de revistas en las que se concentra el mayor interés por publicar los resultados de la investigación y difusión científica por parte de investigadores e instituciones.

Por lo mismo, los títulos indizados en ISI y Scopus son los de mayor interés para la comunidad, sin embargo, tienen un costo muy elevado para las instituciones, además de que, por lo general, se tienen que comprar en paquetes. Peor aún, con la gran expansión de Internet a finales de los años noventa, las bibliotecas universitarias se vieron obligadas a invertir una parte importante de su presupuesto anual en la compra de revistas en formato electrónico, que en un principio se incluían sin costo con los títulos impresos, pero pronto se comercializaron en forma independiente, con precios cada vez más elevados.

Cabe señalar que, anualmente, se producen casi dos millones y medio de artículos científicos, con lo cual es prácticamente imposible que una biblioteca o consorcio pueda adquirir y gestionar todo lo que se produce. 
En otro sentido, a principios de los setenta, los precios de las revistas científicas eran razonables y los aumentos cada año eran pequeños; sin embargo, con la emergencia de la Big Science (Albornoz, 2007: 57) y el crecimiento acelerado de la oferta de educación superior y posgrados en la década de los noventa, la investigación científica adquirió una posición estratégica, a la vez que empezó a tener un desarrollo exponencial a raíz de la explosión tecnológica, con lo cual empezó a darse un incremento desmedido y constante en el número de publicaciones periódicas académicas y científicas y en sus precios.

\section{Un estudio realizado por la Comisión Europea en 2006 expuso que}

la crisis de las revistas hoy en día continúa afectando los presupuestos de las bibliotecas: en los últimos treinta años los precios de las revistas científicas se han incrementado de forma gradual, ejemplo de ello es que entre los años de 1975 y 1995 , este incremento ha sido entre un 200 y un 300 por ciento, muy por encima de la inflación (EC, 2006: 5).

Ante este panorama, los editores comerciales detectaron un potencial negocio y empezaron a obtener los derechos de producir, distribuir y gestionar revistas de terceros, organizándolas en paquetes y bases de datos, e impusieron sus modelos de precios (Anglada y Comellas, 2002). Al mismo tiempo, algunas sociedades científicas empezaron a explotar el valor comercial de sus propias revistas, de tal suerte que la mayor parte de estas publicaciones generó sus propias plataformas digitales.

Como resultado de este fenómeno, las comunidades científicas y académicas empezaron a impulsar distintos proyectos de acceso abierto para las revistas especializadas. Si bien las principales declaraciones del OA se han dado en Europa, el movimiento de acceso abierto en América Latina es muy importante. Por ejemplo, en 1998 se creó en Brasil la iniciativa de la hemeroteca Sciencific Electronic Library Online (SciELO) para incluir publicaciones científicas de alta calidad en texto completo de América Latina en AA.

Son muchos los factores que han impactado en el desarrollo del movimiento de acceso abierto a la producción científica, puesto que "durante los últimos veinte años la publicación científica ha estado en el centro de grandes transformaciones y cambios potenciados por la revolución digital" (Sánchez-Tarrago, 2016: 159). En cuanto a los aspectos generales, podemos hablar de dos vertientes: 1) la transición de la revista impresa a electrónica y la coexistencia de ambos formatos y 2) la inquietud creciente de investigadores e instituciones por dar mayor visibilidad y difusión a la producción científica. En cuanto a los elementos particulares, cabe mencionar los siguientes: 
- Para los investigadores: la expansión de Internet facilitó la distribución y acceso a contenidos remotos de forma inmediata y, al mismo tiempo, las posibilidades que ofrece la tecnología de hacer más visibles los trabajos y multiplicar las posibilidades de ser citado, no solamente por publicar en un determinado grupo de revistas selectas, sino por el propio contenido de un artículo, lo cual generó nuevas expectativas más allá de las establecidas por el ISI y Scopus.

- Para los revisores investigadores: el desarrollo de nuevas tecnologías amplió las posibilidades de acceso a los conjuntos de datos (datasets) en los que están basadas las investigaciones, además de brindar la posibilidad de articular procesos de revisión y valoración más transparente que permite ampliar el intercambio de ideas y comentarios, incluso posteriormente a la publicación de un trabajo.

- Para las bibliotecas: la necesidad de liberarse de las continuas presiones por parte de los editores, a causa de los elevados precios y continuos incrementos de las revistas. La inquietud por conformar colecciones digitales con los derechos, al menos sobre los trabajos de los investigadores de la institución a la que pertenecen. El imperativo de garantizar la preservación a largo plazo de los resultados de la investigación de su institución.

- Para las instituciones públicas que financian la investigación: el compromiso con la difusión y evaluación más eficaz y eficiente de los resultados de investigación que permite el crecimiento económico y al mismo tiempo el desarrollo de una sociedad basada en el conocimiento.

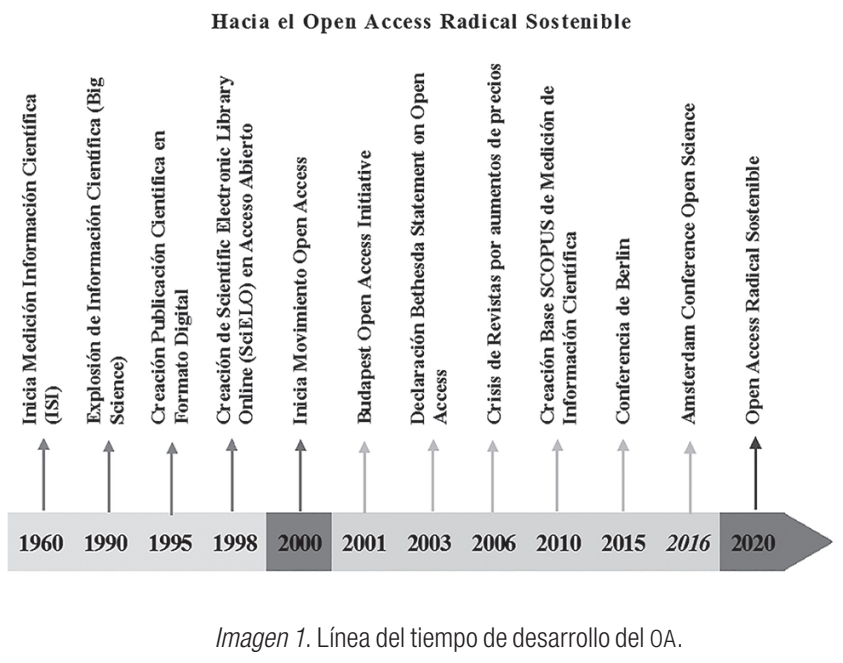




\section{EL MODELO TRADICIONAL DE PUBLICACIÓN CIENTÍFICA EN CRISIS}

Desde el punto de vista de una institución que ha financiado una investigación, parece absurdo que, a través de la biblioteca, tenga que pagar por un artículo que ha producido uno de sus investigadores, o bien que la biblioteca no adquiera la revista y no pueda consultar el artículo, puesto que resultaría muy costoso adquirir todos los títulos en los que publican sus autores.

Desde el punto de vista del investigador, es muy problemático el periodo que tiene que esperar desde que escribe el artículo hasta que se publica, lo cual hace que se vea afectado en sus procesos de evaluación, promociones, reconocimientos y compensaciones, a la vez que el artículo pierde vigencia.

Peor aún, si el autor no publica su artículo en una revista registrada en los índices de reconocimiento internacional, su publicación tendrá poca visibilidad y, por ende, mínimas posibilidades de tener citas, lo que también repercute en sus evaluaciones.

Asimismo, debe referirse la falta de transparencia en todo el proceso de publicación, puesto que no siempre se hacen visibles a los autores las etapas del procedimiento, y muchas veces las editoriales eligen algunos artículos por el renombre del autor o porque pertenece a determinada institución, de tal forma que no todos los artículos son revisados por pares.

\section{EL ACCESO ABIERTO EN LA ACTUALIDAD}

El AA siempre nos remite a artículos científicos o académicos de alta calidad y rigurosa revisión por pares, de tal suerte que se ejecutan a través de los mismos procesos de revisión, producción y publicación de las revistas y libros publicados, bajo el modelo tradicional basado en suscripción. Para que se consideren de acceso abierto, deben estar disponibles gratuitamente y en línea en cualquier momento. En algunos casos, estos artículos pueden depositarse en bases de datos bibliográficas y repositorios institucionales, sin ningún periodo de embargo.

En la actualidad coexisten dos modelos para la publicación de acceso abierto: el de pago por publicar, bajo el control de editores comerciales, y el de instituciones y comunidades de investigadores que se expresa en la Iniciativa 2020 de Acceso Abierto (Conferencia de Berlín, 2015), y propone que las instituciones reorienten sus gastos de suscripciones hacia fondos de acceso 
abierto en los que se puedan financiar los principales servicios que proporcionan los editores, o sea, que esos gastos se orienten a la administración, edición, revisión por pares y difusión, entre otros aspectos. Dentro de los modelos de acceso abierto existe un amplio espectro de posibilidades, por ejemplo, Ruta verde, Ruta dorada, Ruta diamante, Ruta platino, acceso abierto con registro, acceso abierto total, el acceso abierto híbrido, etcétera.

\section{Pago por publicar o Article Processing Charge (APC)}

Con motivo de la fuerza que ha tomado la iniciativa del OA en el medio científico y académico, diferentes editores y proveedores de información científica se han propuesto convertir revistas de suscripción a revistas de acceso abierto o híbridas. De tal forma, como expresó Lluís Anglada (2017): "la concentración de la publicación científica en empresas editoriales comerciales empezó en los años [setenta] del pasado siglo, pero ha llegado a extremos oligopolísticos con Internet", a tal grado que gran parte de la comunidad científica solicita que el "control de las publicaciones científicas vuelva a la academia".

En el pago por publicar, el autor o su institución pagan al editor una cuota para que su artículo pueda consultarse en acceso abierto, las cuotas son altas, de tal suerte que se vuelve más costoso que el pago por suscripción. Por supuesto que quienes se ven más favorecidos son "Los cinco grandes del mercado editorial científico”: Elsevier, Sage, Springer-Nature, Taylor \& Francis y Wiley (ABC Ciencia, 2015).

En el apc tienen ventaja las revistas de disciplinas que reciben subvenciones, mientras que las que reciben poco o nulo apoyo económico están en desventaja, porque no tienen recursos para pagar sus artículos. Al mismo tiempo, se ven favorecidas las publicaciones de los resultados de las investigaciones de los países con mejor situación económica. De tal forma que existe un sesgo importante en la publicación y difusión de los resultados de la productividad científica.

Por otra parte, el hecho de que se tenga que pagar por publicar despierta suspicacias sobre el producto y, al mismo tiempo, sobre la intención tras el hecho de dar un producto gratuito, lo cual incomoda mucho a las comunidades científicas. Aunque, desde luego, se reconoce la importante contribución que han hecho hacia la publicación científica los editores y proveedores comerciales, han generado problemas, puesto que, finalmente, unos cuantos dominan el mercado de las revistas con base en una evaluación científica, relacionada sobre todo con los indicadores de las publicaciones de uno o dos 
proveedores (80 por ciento de las citas están en ISI Web of Knowledge y Scopus de Elsevier).

Por el lado contrario, las comunidades científicas, sobre todo de instituciones públicas, presionan por el AA, con base en el costo real de la comunicación científica y con nuevos procesos de evaluación que no dependan tanto de indicadores bibliométricos y que permitan a los autores conservar los derechos sobre sus obras.

\section{Modalidades de publicaciones de ACCESo Abierto}

Los títulos que responden total o parcialmente al concepto de AA se clasifican en cuatro grupos:

1) Publicaciones que después de un embargo de 6 a 12 meses facilitan el acceso a sus ficheros o los depositan en bases de datos como PubMed Central.

2) Revistas OA, en las que los autores retienen los derechos de autor y pagan por la publicación de sus artículos. Los ejemplos más claros y conocidos son los de BioMed Central (bmc) y las revistas de la Public Library of Science (plos Biology y plos Medicine).

3) Publicaciones de AA en las que el autor no paga por la publicación de sus trabajos. Ejemplo de este tipo de título se almacenan en el Directory of Open Access Journal (DOAJ), creado y administrado por la Universidad de Lund o la Hemeroteca SciELO.

4) Modelo de pago por publicar, que presenta dos tipos de revistas: de AA totalmente e híbridas, que tienen artículos de suscripción y de pago que están en AA. Este modelo está en amplia expansión por parte de la mayoría de los editores.

Cada editor comercial ofrece en su sitio web un listado de todos sus títulos, en el entendido de que sólo hay acceso al texto completo de las revistas que se suscriben y a aquellas en que expresamente se señalan como de acceso abierto; sin embargo, en el caso de las de acceso híbrido no se especifica, de tal forma que se localizan de manera individual los artículos de acceso abierto que fueron pagados por los autores y que pueden consultarse sin tener suscripción, pero solamente si el usuario sabe de antemano que son de acceso abierto, pues están incluidos dentro de revistas que son de suscripción. 
Una de las primeras editoriales en poner este modelo en práctica fue Springer, que creó el programa Open Choice. Cada editor personaliza su plataforma y presenta esta información en forma muy variada, al igual que los costos para los autores que quieran publicar sus artículos en revistas de acceso abierto o híbridas. Prácticamente todos los editores han incluido en sus colecciones artículos de pago por publicar, tanto en títulos OA, como en títulos híbridos.

A manera de ejemplo del costo de pago por publicar, mostramos enseguida una relación de los principales editores con los precios en dólares, con base en información del 2007 presentada por Melero y Abad (2008).

\begin{tabular}{|l|c|r|c|}
\hline \multicolumn{1}{|c|}{ Editorial } & Programa OA hibrido & $\begin{array}{c}\text { Precio/artículo } \\
\text { USD }\end{array}$ & Copyright \\
\hline American Chemical Society & ACS autor choice & $1,000-3,000^{*}$ & Autor \\
\hline American Physical Society & Free to read & $975-1,300$ & Editorial \\
\hline Blackwell Publishing & Online open & 2,600 & Autor \\
\hline bmi Publishing Group Ltd. & BMJ unlocked & 3,145 & Editorial \\
\hline Cambridge University Press & Cambridge open option & 2,700 & Editorial \\
\hline Elsevier & Sponsored payment & 3,000 & Editorial \\
\hline HighWire Press & Author-side payment & $500-3,500$ & CC Licence \\
\hline John Wiley and Sons & Funded access & 3,000 & Editorial \\
\hline Oxford University Press & Oxford open & $1,500-2,800^{*}$ & CC Licence \\
\hline Royal Society & EXIS open choice & $370-550$ page & Autor \\
\hline Royal Society of Chemistry & RSC open science & $1,000-2,500$ & Editorial \\
& & $($ Euros) & \\
\hline Springer & Open choice & 3,000 & CC Licence \\
\hline Taylor and Francis & Open access & 3,100 & CC Licence \\
\hline National Academy of Sciences & Open Access fee & $1,100^{*}$ & CC Licence \\
\hline
\end{tabular}

Cuadro 1. Costo por artículo de acceso abierto en revistas en 2007.

* Depende del tipo de vínculo con la sociedad cientííca.

Las cuotas actuales son similares, con excepción de American Chemical Society que ha tenido un aumento sustancial, ya que pasó de 1000/3000 dólares a 2000/4000 dólares. 
Se trata de una iniciativa avalada por más de 560 instituciones con base en la Declaración de Berlín sobre Acceso Abierto al Conocimiento en Ciencias y Humanidades (Sociedad Max Planck, 2003), cuyo objetivo es acelerar la transición al acceso abierto mediante la transformación del actual modelo comercial de suscripción de revistas científicas, a un acceso abierto total. Los principios de esta iniciativa se discutieron y acordaron en la Conferencia de Berlín (2015).

A partir de entonces, se ha generado una gran discusión y reflexión sobre las características del AA como práctica, puesto que tiene que pasar por la transformación del modelo de suscripción que representa un negocio muy lucrativo para algunos editores y proveedores de información especializada.

Se busca generar una transformación planificada del sistema editorial, por medio de modelos que garanticen que "los productos sean abiertos y reutilizables y que los costos detrás de su difusión sean transparentes y económicamente sostenibles" (Sociedad Max Planck, 2003).

La iniciativa reconoce y apoya distintas formas de implementar el AA, incluso el desarrollo de nuevas plataformas de publicación de OA, archivos y repositorios. Lo importante es propiciar una transición fluida y ágil, con orientación académica, con la finalidad de que todo el corpus existente de revistas académicas deje de ser de suscripción y se convierta plenamente en acceso abierto.

Esta iniciativa se contempla como un elemento de una evolución más profunda del sistema de publicación académica, que logre conducir a mejoras importantes en la comunicación académica y la evaluación de la investigación. Es más, se expresa que los últimos avances y estudios indican que este proceso de transición puede realizarse en el marco de los recursos actualmente disponibles. Para lograr esta transición se tomaron los siguientes acuerdos:

1) Transformar la mayoría de las publicaciones académicas de suscripción a acceso abierto, de acuerdo con las preferencias de publicación específicas de cada comunidad.

2) Invertir los recursos que actualmente se destinan a suscripciones en fondos para respaldar modelos de negocio sostenibles de OA. Es decir, reorganizar los flujos de efectivo subyacentes, establecer transparencia respecto de los costos y posibles ahorros, y adoptar mecanismos para evitar barreras indebidas a la publicación. 
3) Invitar a todas las partes involucradas en publicaciones académicas, en particular universidades, instituciones de investigación, patrocinadores, bibliotecas y editores, a colaborar en una transición rápida y eficiente hacia el OA total.

4) Establecer en una hoja de ruta los pasos e hitos específicos para el proceso de transformación.

En este contexto, se han hecho diversas declaraciones posteriores; por ejemplo, en la Declaración de Amsterdam (Amsterdam Conference Open Science, 2016), se establece que el movimiento de AA a las publicaciones científicas se encuentra en una encrucijada. Sin embargo, después de varios años de una difícil lucha para convencer a actores bastante escépticos, ahora disfruta de un poderoso apoyo y, según se expresa: "La importancia del acceso abierto ya no es un tema de discusión" (Bibliothèque Scientifique Numérique, 2016). En este tenor, el 27 de mayo de 2016, en una reunión de Consejo de la Unión Europea sobre la transición hacia una ciencia abierta en Bruselas, se acordó que, dado que los resultados de la investigación científica son un bien público, deben estar a disposición de todo público sin costo alguno. La discusión sigue en diversas entidades y foros, y al mismo tiempo la confrontación entre universidades y editores comerciales es cada vez más abierta y hostil.

\section{ANÁLISIS Y PROPUESTAS}

A partir de lo anterior, señalamos que el movimiento OA está en expansión y ha alcanzado un alto grado de legitimidad, pues se ha convertido en un modelo alternativo al esquema hegemónico de difusión de información científica. Cabe subrayar que América Latina ha sido vanguardia al respecto, pues desde comienzos del siglo XXI conformó hemerotecas robustas, como SciELO, Redalyc y Latindex, las cuales responden completamente a la noción de acceso abierto y fueron creadas para facilitar la difusión y acceso al conocimiento científico.

Indudablemente, las iniciativas de acceso abierto se han generado como una respuesta a la concentración de poder de las empresas editoras/proveedoras de información científica, las cuales han sido señaladas como parte de una oligarquía académica. Por lo tanto, se trata de un asunto primordialmente económico, pues las instituciones científico-académicas se encontraban "atrapadas en un sistema en el que, además de subsidiar los procesos de investigación, deben pagar el acceso a fuentes de información publicadas 
por editoriales que, paradójicamente, se constituyen en depositarias de los derechos de propiedad intelectual de buena parte de los resultados de su actividad" (Molfino y González, 2012: 2).

La iniciativa OA es de muy amplio espectro, pues si bien originalmente surgió como una oposición a las restricciones económicas del esquema tradicional, pronto asumió la responsabilidad de incluir la revisión por pares (peer review) como mecanismo fundamental de una evaluación capaz de garantizar la calidad de las publicaciones, con base en los estándares de comunicación científica. Por lo mismo, ha planteado la necesidad de repensar el esquema, ya que la revisión "doble ciego" no necesariamente garantiza una evaluación justa ni, una vez aceptada la publicación, la oferta de la mejor información científica posible.

En este tenor, surgió la propuesta de la revisión abierta (Open Review, OR), que implica la transparencia en el proceso de la revisión, pues autores y revisores conocen sus respectivas identidades, además, impulsa la colaboración entre ellos y, al mismo tiempo, con los editores de la revista, bajo un principio distinto al de la competencia que fomenta el doble ciego y resulta elitista, todo ello con la idea de entre todos construir un artículo mucho mejor acabado.

Además, se trata de un asunto político-epistémico, pues el movimiento OA es una respuesta crítica y una alternativa al modelo hegemónico de acopio y difusión de información científica, concebido de manera mercantil en términos de distribución y colonial en cuanto a la producción y valoración de los conocimientos, ya que dicho modelo ha configurado, según Guédon (2011), una división tajante entre "ciencia principal" y "ciencia periférica". Por lo mismo, el movimiento de apertura de información y conocimiento reconoció el sesgo geográfico y lingüístico producido por el esquema dominante y la necesidad de visibilizar la producción científica de los países periféricos, sobre todo en el contexto de la propuesta de construcción de una sociedad mundial del conocimiento.

Asimismo, el movimiento del OA ha respondido a la necesidad de combatir el cercamiento/privatización de los recursos de información/conocimiento que se ha producido en el capitalismo tardío (postindustrial e informacional), por medio precisamente de las cinco empresas principales que controlan el mundo de la información científica (ABC Ciencia, 2015), que en su afán de rentabilidad llevaron al límite a las instituciones de investigación/ educación, principales demandantes de información especializada, quienes comenzaron a admitir que ya no podían solventar los altos costos de las suscripciones a los recursos de dichas empresas. Por tal razón, en años recientes, en los países desarrollados se han comenzado a impulsar iniciativas orientadas a afianzar (estructural y globalmente) el modelo de acceso abierto para sus publicaciones. 
De igual modo, y para profundizar en la cuestión política, nos parece insoslayable cuestionar la concepción misma de propiedad intelectual. Es decir, no se puede estar a favor del movimiento del OA e ignorar las cuestiones de derecho de autor. Lander (2001: 6), desde la óptica decolonial — que pone en evidencia la división norte/sur que existe en nuestro mundo-, nos recuerda que en el orden en que vivimos los derechos de propiedad intelectual "corresponden exclusivamente a las modalidades universitarias/empresariales de los regímenes del saber occidental y, por lo tanto, es la protección de una propiedad intelectual que es individual y es concebida como derecho privado”. Es decir, en esa noción dominante de derecho de autor, el conocimiento es definido como un producto individual y que, por lo tanto, debe protegerse de manera particular. Lo que queremos señalar es que hay otras maneras de entender la información y el conocimiento.

En ese orden de ideas, nos encontramos ante un fenómeno de carácter económico, social, político y epistémico, que nos conduce a la pregunta: ¿a quién le pertenece el conocimiento? Por ello, es pertinente poner sobre la mesa las discusiones más amplias que se han dado a nivel mundial sobre la posibilidad de elaborar otros patrones de producción, evaluación, distribución, consumo y propiedad del conocimiento.

Conviene recordar que la iniciativa del OA forma parte de un movimiento más extenso, denominado cultura libre, el cual básicamente tiene que ver con una concepción del conocimiento y la información, no de manera individual y mercantil, sino comunitaria.

La cultura libre ha influido en distintos ámbitos sociales y académicos. En la disciplina bibliotecológica se acuñó la noción de bienes comunes de información (BCI) (Kranich, 2004), que parte justo de la idea de comunidades (virtuales) de información, y de Internet como dominio público. Los BCI, como recursos de información abiertos y democráticos, tienen los siguientes rasgos: acceso libre y abierto, autogobernanza, colaboración, gratuidad o bajo costo y sustentabilidad.

Asimismo, la cultura libre promueve el modelo denominado "procomún”, que se ha definido como "un sistema social que relaciona íntimamente a las personas o partes interesadas con sus recursos y con las formas participativas en las que los gestionan/producen y cuidan de ellos" (Kranich, 2004).

Desde esta perspectiva, la información y el conocimiento (y la tecnología misma) no son concebidos exactamente como recursos, privados (del mercado) o públicos (del Estado), sino como bienes comunes, que nos pertenecen a todos porque son producto de la Inteligencia Colectiva. Aquí tendríamos la respuesta a la pregunta anterior. Diríamos que el conocimiento no le pertenece a nadie en particular, sino a todos. Por tal razón, su propiedad 
y manejo debiera ser igualmente común o comunal, y así responder a un esquema libre —en términos de derecho de autor - y de autogobernanza —en cuanto a la gestión-, que implique su circulación libre, abierta y gratuita, para lo cual se requieren prácticas colaborativas, en el caso del conocimiento científico: entre autores, editores, revisores y bibliotecarios. Todo lo anterior, responde por completo a la esencia del OA y a la idea de BCI, concebidos así por Kranich (2004).

Ahora bien, uno de los principales retos del modelo del OA es la sostenibilidad, la capacidad de mantener su producción a futuro. En primer lugar, diríamos que para que una iniciativa de esta índole sea sostenible, debemos considerar integralmente aspectos económicos, tecnológicos y jurídicos; principalmente económicos porque, como dijimos, la edición implica costos - aunque sean significativamente más reducidos en la modalidad digital de lo que eran en la impresa-; tecnológicos porque se requiere el uso de hardware y software para la edición y publicación; y jurídicos, en principio por la cuestión de la propiedad intelectual, así como por la necesidad de establecer leyes que respalden políticas de información sobre el OA.

Y más allá de estas cuestiones que implican un papel protagónico de las instituciones públicas, porque es evidente que la mayor parte de la investigación científica es financiada por aquéllas y porque el marco jurídico pertinente les compete, nos parece sugerente pensar de manera más amplia y un poco diferente el asunto de la gestión de la información especializada.

Dentro de la cultura libre podemos identificar cuatro tipos de iniciativas que se vinculan (real o potencialmente) con el acceso abierto. La primera es la propuesta de software libre, cuyo pionero es Richard Stallman, informático y activista estadounidense que se opuso al esquema privativo de los recursos informáticos y a que se mantuviera cerrado el código fuente. Éste fue el primer proyecto en cuestionar la lógica mercantil de la información, en el avance de la computación y la amenaza de un modelo que limitaba el derecho a la información y el conocimiento.

La segunda es la más claramente relacionada con el OA y tiene que ver con las licencias libres en materia de derechos de autor, cuyo ejemplo más ampliamente conocido es Creative Commons, un modelo desarrollado por el abogado estadounidense Lawrence Lessig, quien a su vez se inspiró en el esquema de propiedad intelectual desarrollado previamente en el mundo de la informática y por el propio Stallman, denominado General Public License (GPL).

La tercera iniciativa que nos gustaría presentar es el paradigma Peer to Peer (P2P) hacia una red de pares o red entre iguales, que implica una forma distribuida (no jerárquica) y descentralizada de intercambio de información. 
La cuarta iniciativa es el formato wiki, el cual refiere la construcción colaborativa del conocimiento y sus consiguientes recursos de información.

En este tenor, podemos decir que el software libre es la piedra angular de la cultura libre y, por tanto, es condición sine qua non para el acceso abierto radical y sostenible, puesto que, como señalan Torres y Zurita:

Para poder brindar un acceso a la información digital a nivel mundial, necesariamente hay que contar con la infraestructura de hardware y software capaz de soportar un tráfico importante de información a bajo costo, con posibilidades promisorias de crecimiento social y un flujo horizontal de los datos, por lo que el movimiento de software libre basado en el fenómeno de gnu/Linux se yergue como una sólida alternativa para crear condiciones de carácter tecnológico y cultural que den salida a los problemas de acceso a la información más apremiantes de nuestros días (Torres y Zurita, 2007: 144).

Entonces, el OA será sostenible en la medida que forme parte de otras iniciativas de cultura libre, por dos razones: 1) el OA debe concebirse como parte de un movimiento de liberación del conocimiento, y de hecho constituye una suerte de activismo académico; 2) los principios de todas estas manifestaciones de la cultura libre tienen todo que ver con el ethos científico de Merton (1942), particularmente con los principios de comunismo (los científicos renuncian a la propiedad intelectual a cambio de reconocimiento) y de desinterés (no hay más interés que contribuir al conocimiento).

Lo anterior significa que el OA asumiría, además de las licencias libres, el uso de software libre para todo el trabajo de edición y publicación (lo que reduce significativamente los costos), y que sería conveniente implementar una estructura P2P a nivel global de gestión de información científica, que combatiera su centralización, todo ello en el más puro espíritu wiki de producción, evaluación, edición, revisión y publicación.

Lo que hemos señalado en este análisis responde a una perspectiva comunal de la información y el conocimiento, razón por la cual se propuso la noción de procomún para referir una alternativa a la gestión de aquéllos. Al respecto, debemos señalar que lo común implica la superación de la dicotomía público/privado, entre el Estado y el mercado como únicas instancias (a veces excluyentes, a veces cooperantes) legitimadas para administrar los recursos (naturales o culturales), no obstante, el riesgo de centralización y privatización que suponen. Lo común sugiere una tercera vía, en la que la ciudadanía se haría cargo. Pero, dado que el Estado y el mercado tienen un papel preponderante en la organización social (incluido el campo científico) cabe la pregunta, ¿el esquema comunal es compatible con el Estado y el mercado? 
De acuerdo con Bollier (2016: 136), "casi todos los comunes son híbridos que en alguna medida dependen del Estado o del mercado" Por lo tanto, sí existiría una cooperación entre los comunes y el Estado, el cual, con el apoyo de las instituciones académicas, debería incentivar la gestión ciudadana de los recursos, pues ha de admitir que para él es imposible hacerlo de manera adecuada (justa, eficiente y transparente), y que, en todo caso, debe velar por el interés común. Igualmente, Bollier nos recuerda que mercado no es exactamente lo mismo que capitalismo, así que "el mercado puede ser completamente local, justo y sensible a las necesidades de la colectividad, siempre y cuando esté lo suficientemente integrado en la comunidad y dependa de ella" (ibíd.: 136).

\section{CONSIDERACIONES Finales}

Si bien el movimiento del OA ha cobrado fuerza en los últimos años, su trayectoria es breve y aún tendrá que enfrentar diversos retos, pues el problema es complejo e incluye distintos actores y temáticas.

El OA reivindica el acceso gratuito a la información científica, sin embargo, sabemos que la edición de las revistas científicas genera gastos que deben ser cubiertos, y que para el mantenimiento de un alto nivel de calidad se demanda un grado de dedicación y profesionalismo que debe retribuirse y, por lo tanto, se requiere desarrollar un factor de sostenibilidad en el contexto de la cultura libre.

Algunas universidades de países desarrollados trabajan con las editoriales sobre nuevos modelos, y con ello se abren nuevas perspectivas para el OA en el corto plazo, por lo cual es muy importante estar atentos a esta evolución, ya que este movimiento incide de manera importante en bibliotecas, editores, científicos, universidades y usuarios de la información especializada.

A lo largo del proceso de la transición de publicaciones periódicas impresas a formato electrónico, las instituciones académicas permitieron que los empresarios editoriales impusieran sus modelos de precios. Asimismo, han aceptado la tendencia actual de pago por publicar, sin embargo, es momento de revisar los modelos de precios.

El APS ha tenido éxito, ya que se incrementa la difusión de los artículos y sus citas. No obstante, no existen políticas claras institucionales, no se ha establecido quién tiene que pagar. En ocasiones, los autores inscriben proyectos para adquirir financiamientos y poder pagar para publicar. En algunos casos, se ha planteado que las bibliotecas asumirían estos gastos, en la 
medida que el AA libere recursos antaño dedicados al pago de suscripciones. De hecho, en el contexto actual se ha pasado de un modelo de pagar por consultar, a pagar por publicar, con lo cual, al final, las instituciones educativas realizan un pago doble por los contenidos científicos, puesto que cada vez más las revistas suscritas son híbridas, es decir, contienen artículos pagados por los autores.

Consideramos que las instituciones académicas, sobre todo las públicas, deben oponerse al pago por publicar y apoyar ampliamente las iniciativas del OA radical, que sólo se logrará en la medida en que cambie el modelo de gestión de las actuales revistas académicas y se erradique el sistema de suscripciones comerciales. Los nuevos modelos habrán de adaptarse a los propósitos de la ciencia abierta y la cultura libre, encausados a transformar el medio editorial, de tal forma que se evite la dominación sobre el conocimiento científico de un pequeño grupo de editores.

De hecho, nos encontramos ante un fenómeno de carácter económico, jurídico, social, político y epistémico; por ello es pertinente promover discusiones amplias para definir otros patrones de producción, evaluación, distribución, consumo y propiedad del conocimiento.

La esencia del OA demanda que se contemple la circulación libre, abierta y gratuita del conocimiento científico, en un contexto de prácticas colaborativas entre autores, editores, revisores y bibliotecarios, puesto que debemos recordar que la ciencia nació como una práctica abierta, colaborativa y desinteresada.

El derecho de acceso a la información incluye el derecho de acceso a la información científica y, en este sentido, académicos y científicos debemos ser custodios y garantes de este derecho, en virtud de que el conocimiento es un bien común. Por lo mismo, es necesario que todos los actores se involucren para apoyar una transición fluida, ágil, con orientación académica, con la finalidad de que todo el corpus existente de revistas académicas deje de ser de suscripción y se convierta plenamente en acceso abierto.

En resumen, se sugiere un esquema que garantice el derecho de acceso a la información científica, así como su calidad y sostenibilidad, en una dinámica de apertura y colaboración, tanto en la producción, como en la revisión, edición y publicación, y con un nuevo marco jurídico que incluya políticas de información y otras nociones de propiedad intelectual. 


\section{REFERENCIAS}

ABC Ciencia. 2015. "Estas cinco editoriales controlan más de la mitad de las publicaciones científicas desde 2006”, en <http:/www.abc.es/ciencia/20150612/abci-control-publicaciones-cientificas-2015061209-43html>, consultada el 5 de octubre de 2018.

Albornoz, Mario. 2007. "Los problemas de la ciencia y el poder". Revista Iberoamericana de Ciencia, Tecnología y Sociedad 8 (3): 47-65.

Amsterdam Conference Open Science. 2016. "Amsterdam call for action en Open Science". Ámsterdam: ponencia presentada en la "Amsterdam Conference Open Science. From Vision to Action”, 4-5 de abril, en <https://web.archive.org/ web/20170202044907/https:/www.eu2016.nl/documenten/rapporten/2016/04/ 04/amsterdam-call-for-action-on-open-science >, consultada el 4 de octubre de 2018.

Anglada, Lluís. 2017. "Cuando se jodió lo nuestro o De la devolución de los contenidos académicos a los académicos". Block de Bid, 18 de octubre, en <http://www.ub.edu/ blokdebid/es/content/cuando-se-jodio-lo-nuestro-o-de-la-devolucion-de-los-contenidos-academicos-los-academicos>, consultada el 4 de octubre de 2018.

Anglada, Lluís y Núria Comellas. 2002. “¿Qué es justo? Modelos de precios en la era electrónica”. Library Management 23 (4-5): 227-233.

Bibliothèque Scientifique Numérique. 2016. "Tous les articles scientifiques européens en libre accès à partir de 2020". IST de la BSN, 30 de mayo, en <http:// www.bibliothequescientifiquenumerique.fr/tous-les-articles-scientifiques-europeens-en-libre-acces-a-partir-de-2020/>, consultada el 5 de octubre de 2018.

Bollier, David. 2016. Pensar desde los comunes. Madrid: Traficantes de Sueños.

Conferencia de Berlín. 2015. "Open Access 2020: iniciativa para la transformación de las revistas científicas en revistas de Acceso Abierto”. Berlín: Conferencia, 8-9 de diciembre, en <https://oa2020.org/>, consultada el 23 de septiembre de 2018.

European Commission (EC) 2006. "Study on the Economic and Technical Evolution of the Scientific Publication Markets in Europe”. Bruselas: European Commission, Directorate-General for Research Information and Communication Unit, 109 pp., en <https://ec.europa.eu/research/openscience/pdf/openaccess/librarians_2006_scientific_pub_study.pdf>, consultada el 8 de septiembre de 2018.

Finidori, Helen. 2013. “¿Qué es el procomún?” (Trad. de Carmen Lozano Bright). Guerrilla Translation, 3 de diciembre, en <http://www.guerrillatranslation. es/2013/12/03/que-es-el-procomun/>, consultada el 7 de septiembre de 2018.

Guédon, J.C. 2011. "El acceso abierto y la división entre ciencia 'principal' y 'periférica”". Crítica y Emancipación, 36: 135-180.

Howard Hughes Medical Institute. 2003. "Bethesda Statement on Open Access Publishing”. Maryland: 11 de abril,en <http://legacy.earlham.edu/ peters/fos/bethesda.htm>, consultada el 10 de abril de 2018.

Kranich, Nancy. 2004. The information commons. A public policy report. Nueva York: Brenan Center for Justice at nyu School of Law, en $<$ https://open.bu.edu/handle/2144/53>, consultada el 7 de septiembre de 2018 .

Lander, E. 2001. "Los derechos de propiedad intelectual en la geopolítica del saber de la sociedad global”. Revista del Centro Andino de Estudios Internacionales (2): 1-28.

Melero, Remedios y Francisca Abad. 2008. "Revista Open Access: características, modelos económicos y tendencias”. Bid Textos Universitaris De Biblioteconomía $i$ Documentación (20): 17 p, en <http://bid.ub.edu/pdf/20meler2.pdf>, consultada el 18 de septiembre de 2018. 
Merton, Robert. 1942. "Science and Technology in a Democratic Order". Journal of Legal and Political Sociology (1): 115-126.

Molfino, M. del R. y C.M. González. 2012. “Acceso Abierto a la literatura científica y a los datos de investigación: escenario de oportunidad para Latinoamérica. II Ethicomp Latinoamérica, Memoria Académica, 8-12 de octubre. Bahía Blanca, Arg.: en <http://www.memoria.fahce.unlp.edu.ar/trab_eventos/ev.2971/ev.2971.pdf>, consultada el 3 de octubre de 2018.

Open Society Institute (OSI). 2001. "Budapest Open Access Initiative”. Budapest: OSI, 1-2 de diciembre, en<http://www.budapestopenaccessinitiative.org >, consultada el 3 de septiembre de 2018.

Sánchez-Tarrago, Nancy. 2016, "Las revistas científicas en América Latina hacia el camino del acceso abierto: un diagnóstico de políticas y estrategias editoriales”. TransInformação 28 (2): 159-172.

Sociedad Max Planck. 2003. "Declaración de Berlín sobre el acceso abierto en ciencias y humanidades”. Berlín: Conferencia sobre acceso abierto, 22 de octubre,en $<$ https:// www.um.es/c/document-library/get_file?uuid=f3736570-bb84-40b3-8a2ea9397ef7ef30\&groupId=793464>, consultada el 23 de septiembre de 2018.

Suber, Peter. 2004. "What is Open Access? An Overview". Washington, D.C.: ALPSP-SSP Meeting, 8 de noviembre,en <https://legacy.earlham.edu/ peters/ fos/2005/01/conference-presentations.html>, consultada el 3 de octubre de 2018.

Torres Vargas, Georgina Araceli y Juan Manuel Zurita Sánchez. 2007. "Software libre y libre acceso a la información: ¿hacia un ciberespacio público?” Documentación de las Ciencias de la Información (30): 135-149.

Para citar este texto:

Araiza Díaz, Verónica, María Esther Ramírez Godoy y Alma Silvia Díaz Escoto. 2019. "El Open Access a debate: entre el pago por publicar y la apertura radical sostenible”. Investigación Bibliotecológica: archivonomía, bibliotecología e información 33 (80): 195-216. http://dx.doi.org/10.22201/iibi.24488321xe.2019.80.58039 